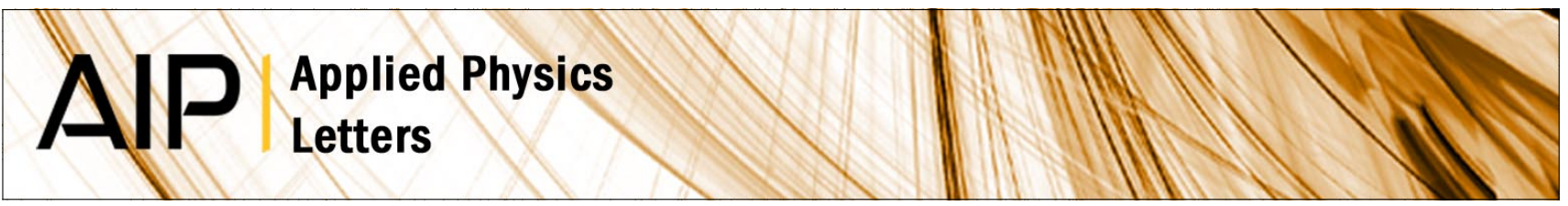

\title{
Enhanced microwave shielding effectiveness of ultrathin pyrolytic carbon films
}

K. Batrakov, P. Kuzhir, S. Maksimenko, A. Paddubskaya, S. Voronovich et al.

Citation: Appl. Phys. Lett. 103, 073117 (2013); doi: 10.1063/1.4818680

View online: http://dx.doi.org/10.1063/1.4818680

View Table of Contents: http://apl.aip.org/resource/1/APPLAB/v103/i7

Published by the AIP Publishing LLC.

Additional information on Appl. Phys. Lett.

Journal Homepage: http://apl.aip.org/

Journal Information: http://apl.aip.org/about/about_the_journal

Top downloads: http://apl.aip.org/features/most_downloaded

Information for Authors: http://apl.aip.org/authors

\section{ADVERTISEMENT}

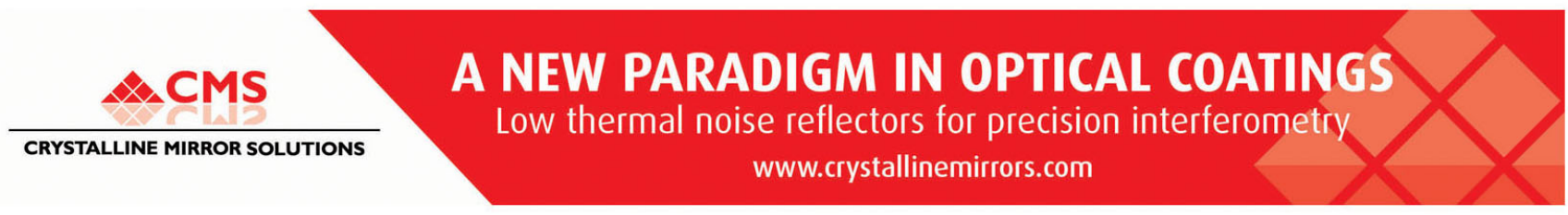




\title{
Enhanced microwave shielding effectiveness of ultrathin pyrolytic carbon films
}

\author{
K. Batrakov, ${ }^{1, a), b)}$ P. Kuzhir, ${ }^{1, b), c)}$ S. Maksimenko, ${ }^{1}$ A. Paddubskaya, ${ }^{1}$ S. Voronovich, ${ }^{1}$ \\ T. Kaplas, ${ }^{2}$ and Yu. Svirko ${ }^{2}$ \\ ${ }^{1}$ Research Institute for Nuclear Problems, Belarusian State University, Minsk 220030, Belarus \\ ${ }^{2}$ Department of Physics and Mathematics, University of Eastern Finland, Joensuu FI-80101, Finland
}

(Received 14 March 2013; accepted 2 August 2013; published online 16 August 2013)

\begin{abstract}
Electromagnetic properties of pyrolytic carbon (PyC) films with thickness from 5 to $241 \mathrm{~nm}$ are studied experimentally and numerically at $28 \mathrm{GHz}$. We observe experimentally that PyC films are capable to absorb up to $50 \%$ of microwave power in the $\mathrm{K}_{\mathrm{a}}$-band. By using boundary conditions in the rectangular waveguide, we demonstrate theoretically that $50 \%$ of microwave power can be absorbed in a conductive film with thickness much smaller than the skin depth. The results of modelling for PyC films on silica substrate are in excellent coincidence with the experimental data. (C) 2013 AIP Publishing LLC. [http://dx.doi.org/10.1063/1.4818680]
\end{abstract}

The overpopulation of the microwave $\mathrm{K}_{\mathrm{a}}$-band that hosts satellite and airborne communication channels has brought attention to a challenging problem of the electromagnetic (EM) compatibility. The hostile electromagnetic environment aboard can strongly affect the performance of the communication devices, which should be protected by EM coatings with high EM interference shielding effectiveness (EMI SE). For satellite and airplane communication systems, the thickness and weight of these coatings became critical issue.

The shielding effectiveness, $S E(d B)=10 \log \left(P_{t} / P_{i}\right),{ }^{1}$ where $P_{t}$ and $P_{i}$ are, respectively, the transmitted and incident electromagnetic power, is determined by reflection and absorption losses. In metal films, the reflection losses occur due to the impedance discontinuity at the film boundaries. They decrease with frequency because metal impedance increases. The absorption losses are determined by the metal skin depth that decreases with the frequency. ${ }^{2}$ It is worth noting that at low temperatures and high frequencies, the conductivity of ultrathin metal film is no longer described by the Drude theory because the thickness of the skin layer is less than the electron mean free path. This leads to anomalous skin effect that manifests itself as anomalously high absorption losses in a metal film whose thickness is a small fraction of the skin depth. ${ }^{3}$

However, it has been demonstrated recently that the anomalous absorption can also be observed at room temperature in microwave frequency range. Specifically, it has been shown both theoretically ${ }^{4}$ and experimentally ${ }^{5}$ that when the EM wavelength is much longer than the skin depth and the film thickness is much smaller than the skin depth, the metal film is capable to absorb up to $50 \%$ radiation at normal incidence. ${ }^{4}$ This phenomenon can be explained by modelling of EM propagation in terms of transmission line replacing the film with a lumped resistor. ${ }^{4}$ In the experiments at the wavelength of $8 \mathrm{~mm},{ }^{5}$ the anomalously high absorption at room

\footnotetext{
a) Also at Physics Department, BSU, Minsk, Belarus.

${ }^{b} \mathrm{~K}$. Batrakov and P. Kuzhir contributed equally to this work.

c)Electronic mail: polina.kuzhir@gmail.com
}

temperatures was observed in aluminium films with thicknesses from 1.7 to $15 \mathrm{~nm}$, i.e., for films several hundred times thinner than the skin depth at this wavelength. It is worth noting that such an anomalous absorption is responsible for damaging windows in powerful microwave and laser systems. $^{6}$

We have recently demonstrated ${ }^{7}$ that $25 \mathrm{~nm}$ thick pyrolytic carbon (PyC) film consisting of randomly oriented and intertwined graphene ribbons, which have typical size of few nanometers, is capable to absorb up to $38 \%$ of the microwave power in the $\mathrm{K}_{\mathrm{a}}$-band. This experimental finding indicates that PyC films can be employed for high EMI SE coatings despite the fact that they are much thinner than the skin depth of metals (e.g., $\mathrm{Al}$ and $\mathrm{Cu}$ ). It has been shown ${ }^{8}$ that sheet resistance of PyC films is close to that of multilayer graphene ${ }^{9,10}$ and carbon nanotubes, ${ }^{11}$ which have already shown outstanding EMI shielding ability. However, in contrast to graphene and carbon nanotubes, PyC films can be grown directly on both dielectric and metallic substrates opening a new route towards fabrication of ultralight and nanometrically thin EMI protective coatings with enhanced shielding effectiveness.

In this paper, we report dependence of the absorptivity and transmittivity of the PyC films deposited on the silica substrate on the film thickness. The measurements were performed at frequency of $28 \mathrm{GHz}$ in rectangular waveguide, which supports the $\mathrm{TE}_{1,0}$ mode. The results of the measurements well correspond to the developed theory of PyC film's EM properties that takes into account the waveguide modes structure.

The PyC films were synthesized on $0.5 \mathrm{~mm}$ thick silica substrates by chemical vapor deposition (CVD), described in more detail elsewhere. ${ }^{8,12}$ Briefly, the CVD chamber was heated to $700{ }^{\circ} \mathrm{C}$ in hydrogen atmosphere $\sim 10$ mbar. At the temperature of $700^{\circ} \mathrm{C}$, the hydrogen atmosphere was replaced by $\mathrm{CH}_{4}-\mathrm{H}_{2}$ gas mixture (the $\mathrm{CH}_{4}-\mathrm{H}_{2}$ ratio and pressure are shown in Table I). Next, in order to start the spontaneous methane decomposition, the CVD chamber was heated up to $1100^{\circ} \mathrm{C}$ by rate of $10^{\circ} \mathrm{C} / \mathrm{min}$. The temperature of the chamber was kept at $1100^{\circ} \mathrm{C}$ for $5 \mathrm{~min}$ and then 
TABLE I. PyC films' synthesis parameters and EMI SE.

\begin{tabular}{lrrccc}
\hline \hline $\begin{array}{l}\text { Thickness } \\
(\mathrm{nm})\end{array}$ & $\begin{array}{c}\mathrm{CH}_{4} \\
(\mathrm{ml})\end{array}$ & $\begin{array}{c}\mathrm{H}_{2} \\
(\mathrm{ml})\end{array}$ & $\begin{array}{c}\text { Pressure at } \\
1100^{\circ} \mathrm{C}(\mathrm{mbar})\end{array}$ & $\begin{array}{c}\text { Pressure at } \\
700^{\circ} \mathrm{C}(\mathrm{mbar})\end{array}$ & $\begin{array}{c}\text { EMI SE } \\
(\mathrm{dB})\end{array}$ \\
\hline 5 & 45 & 20 & 20 & 17.0 & 1.27 \\
9 & 50 & 20 & 22 & 18.0 & 1.91 \\
12 & 60 & 20 & 25 & 20.2 & 2.06 \\
14 & 65 & 20 & 27 & 21.6 & 2.49 \\
18 & 70 & 20 & 29 & 23.4 & 2.84 \\
20 & 75 & 20 & 30 & 24.4 & 3.00 \\
28 & 80 & 20 & 32 & 25.5 & 3.50 \\
30 & 85 & 20 & 34 & 27.1 & 4.11 \\
35 & 90 & 20 & 35 & 27.4 & 4.12 \\
75 & 160 & 0 & 53 & 39 & 6.56 \\
110 & 180 & 0 & 60 & 45 & 7.49 \\
241 & 200 & 0 & 69 & 53 & 7.94 \\
\hline \hline
\end{tabular}

cooled down to $700{ }^{\circ} \mathrm{C}$ during $80 \mathrm{~min}$. At the temperature of $700{ }^{\circ} \mathrm{C}$, the $\mathrm{CH}_{4}-\mathrm{H}_{2}$ gas mixture was replaced by hydrogen. The rest of the cooling was done in hydrogen atmosphere ( $\sim 10$ mbar). It is worth noting that there was no gas flow in the CVD chamber during the deposition process. This allowed us to reduce the gas consumption and also gave more time for poly-aromatic hydrocarbon formation and cross linking. However, the change of the temperature during the deposition process was accompanied with change of the pressure in the CVD chamber. After the CVD process, both sides of the quartz substrate were covered by the PyC film. In order to characterize the film by microwave measurement, one of the substrate surfaces was cleaned out with the harsh oxygen plasma (200 W/20 sccm/3 min).

PyC films' thickness was measured by a stylus profiler (Veeco Instruments, Dektak 150) with an accuracy of $1.5 \mathrm{~nm}$ as well as by atomic force microscope (AFM, Solver P47 PRO, NT-MDT). The structure of the fabricated films was studied by transmission electron microscope TEM JEM-100 $\mathrm{CX}$ at an accelerating voltage of $100 \mathrm{kV}$. The TEM image of the $75 \mathrm{~nm}$ thick PyC film is shown in Fig. 1(a). Raman spectra of the films were measured using Renishaw inVia Raman Microscope at the excitation wavelength of $514 \mathrm{~nm}$. One can observe from Fig. 1(b) that the pronounced $G$ and D peaks at $1582 \mathrm{~cm}^{-1}$ and $1360 \mathrm{~cm}^{-1}$, respectively, dominate the Raman spectrum. The high $I(D) / I(G)$ ratio indicates that the size of the graphene ribbons that compose the PyC film is less than $5 \mathrm{~nm} .{ }^{13,14}$ The fitting of the Raman spectrum revealed strong $D^{\prime}$ and $D^{\prime \prime}$ peaks (see green solid lines in Fig. 1(b)) that show the presence of the amorphous carbon. ${ }^{15-17}$
The microwave measurements were made using a scalar network analyzer R2-408R (ELMIKA, Vilnius, Lithuania), including sweep generator, waveguide reflectometer, and indicator unit (personal computer). The IEC 62431:2008(E) standard specifying the measurement method for the reflectivity of EM materials was used. The EM response of the PyC film as ratios of transmitted/input $\left(S_{21}\right)$ and reflected/ input $\left(S_{11}\right)$ signals has been measured at $28 \mathrm{GHz}$. The scheme of waveguide measurements is presented in Fig. 2. The frequency stability of the oscillator was controlled by frequency meter and was as high as $10^{-6}$. The power stabilization was provided on the level of $7.0 \mathrm{~mW} \pm 10 \mu \mathrm{W}$. Measurement range of EM attenuation was from 0 to $-40 \mathrm{~dB}$. Measurement error of the EM attenuation was $7 \%$. The lateral dimensions of the PyC film were $7.2 \mathrm{~mm}$ $\times 3.4 \mathrm{~mm}$, i.e., the film was deposited on the silica substrate that fits precisely the waveguide cross-section. S-parameters were measured by subsequent insertion of the specimen into the waveguide.

Experimental data for transmittivity $T=S_{21}^{2}$ and absorptivity $A=1-S_{11}^{2}-S_{21}^{2}$ are presented in Fig. 3 . The corresponding data on EMI SE are given in Table I for all samples. The EMI SE provided by the substrate is $1.1 \mathrm{~dB}$. Since the reflectivity and absorptivity of a bare silica substrate are $20 \%$ and $0 \%$, respectively; the substrate contribution dominates the reflected signal, while absorption losses are due to the presence of the PyC film.

One can see from Fig. 3 that in the PyC films with thickness lower than $75 \mathrm{~nm}$, the absorption increases significantly with the thickness. The ability of the film to absorb microwave radiation reaches maximum when the film thickness is $75 \mathrm{~nm}$. Specifically, the $75 \mathrm{~nm}$ thick PyC film is capable to absorb $50 \%$ of the incident power, while 110 and $241 \mathrm{~nm}$ thick PyC films absorb $49 \%$ of incident power. Reflectivity of PyC films of relatively small thicknesses (up to $28 \mathrm{~nm}$ ) is almost the same as for bare quartz substrate (these films reflect 20\%-22\%). Films with thickness higher than $30 \mathrm{~nm}$ reflect from $27 \%$ to $34 \%$ of $\mathrm{EM}$ radiation. As a result, the thickest PyC films demonstrate significant EMI SE. Specifically, only $22 \%, 18 \%$, and $16 \%$ of microwave signal could penetrate through the $\mathrm{PyC}$ film with thicknesses of $75 \mathrm{~nm}, 110 \mathrm{~nm}$, and $241 \mathrm{~nm}$, respectively, deposited on silica substrate. Even 30-35 nm thick PyC films secure already about $60 \%$ of EM attenuation at $28 \mathrm{GHz}$ mostly due to absorption of the microwave radiation. Thus, fabricated $\mathrm{PyC}$ films, whose thickness is thousand times less than the skin depth of conventional metals, provide a reasonably high EM
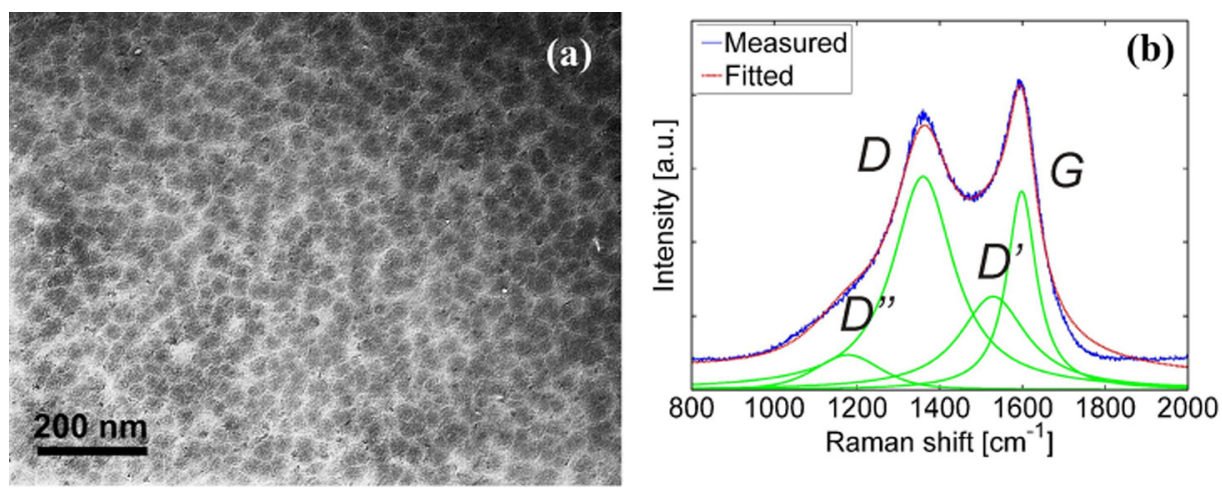

FIG. 1. (a) TEM image of the $75 \mathrm{~nm}$ thick PyC film. (b) Raman spectrum of the PyC film. Blue and red lines show the measured spectrum and results of fitting, respectively. Green lines represent fitting results for the $\mathrm{G}$ (graphite), D (disorder), $D^{\prime}$ (graphite lattice defects), and $D^{\prime \prime}$ (amorphose carbon) resonances. 


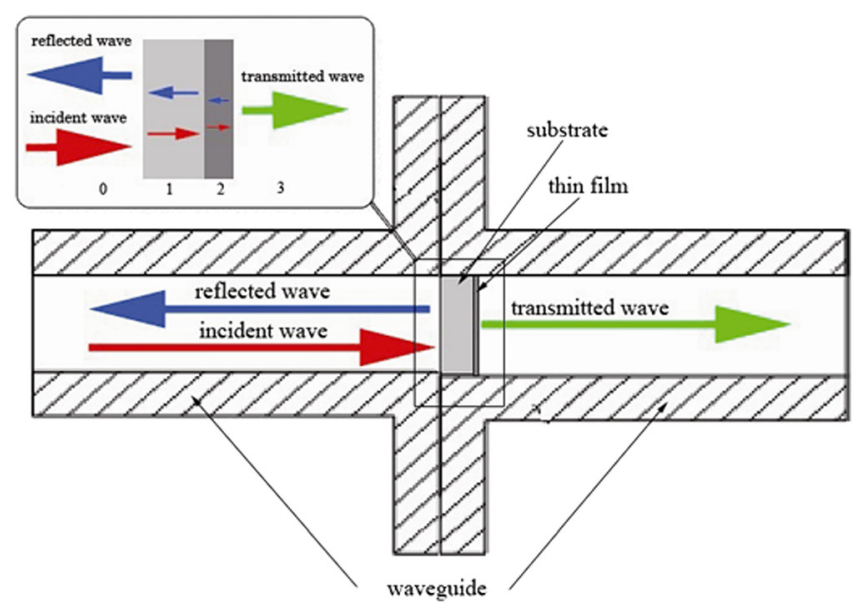

FIG. 2. The scheme of waveguide measurements.

attenuation. The EMI SE of these films is compatible with that for $1 \mathrm{~mm}$ thick polymer composites filled with 2-5 wt. \% of carbon nanotubes (see Refs. 11 and 18 and references therein).

In order to describe the obtained results, we calculated transmittivity and reflectivity of the PyC film in experimental configuration shown in Fig. 2. The longitudinal component of magnetic field associated with the $\mathrm{TE}_{n, m}$ waveguide modes of the vacuum/substrate/PyC film/vacuum system is taken in the form

$$
H_{z i}=i b_{i}^{ \pm} \cos \left(k_{x} x\right) \cos \left(k_{y} y\right) \exp \left( \pm K_{i} z\right) .
$$

The sign \pm corresponds to the counter propagating modes, subscripts $i=0,1,2,3$ label the medium (see Fig. 2), $x, y$ are cross sectional coordinate of the rectangular waveguide, $z$ is the waveguide axis, $k_{x}=\pi n / a, k_{y}=\pi m / b$, where $a, b$ are the waveguide dimensions, $n, m$ are integer, $K_{i}$ $=\sqrt{k^{2} \varepsilon_{i}-(\pi n / a)^{2}-(\pi m / b)^{2}}$ is the wavenumber of the $i$ th medium, the coefficients $b_{i}^{ \pm}$can be found from the boundary

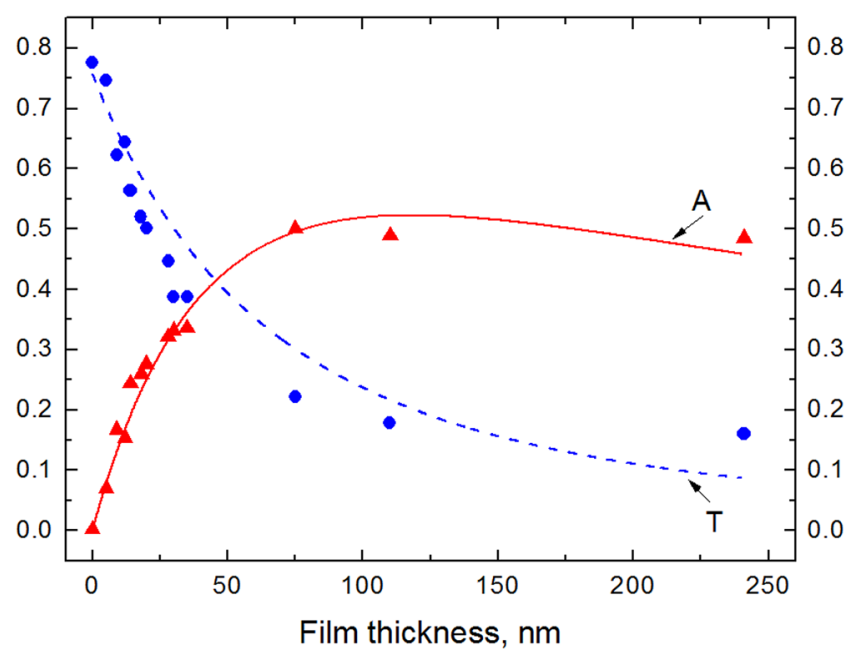

FIG. 3. The dependence of absorption and transmission on PyC film thickness. Triangles and circles correspond to experimental data at $28 \mathrm{GHz}$. Solid and dashed lines give $A$ and $T$, respectively, obtained from numerical solution of Eq. (2) at substrate thickness of $l_{1}=0.5 \mathrm{~mm}$ and the PyC film conductivity of $\sigma=3.5 \times 10^{14} \mathrm{~s}^{-1}$. conditions of tangential electric and magnetic field continuity, which have the following form

$$
\begin{aligned}
b_{0}^{+}+b_{0}^{-} & =b_{1}^{+}+b_{1}^{-}, \\
K_{0}\left(b_{0}^{+}-b_{0}^{-}\right) & =K_{1}\left(b_{1}^{+}-b_{1}^{-}\right), \\
b_{1}^{+} \exp \left(i K_{1} l_{1}\right)+b_{1}^{-} \exp \left(-i K_{1} l_{1}\right) & =b_{2}^{+}+b_{2}^{-}, \\
K_{1}\left[b_{1}^{+} \exp \left(i K_{1} l_{1}\right)-b_{1}^{-} \exp \left(-i K_{1} l_{1}\right)\right] & =K_{2}\left(b_{2}^{+}-b_{2}^{-}\right), \\
b_{2}^{+} \exp \left(i K_{2} l_{2}\right)+b_{2}^{-} \exp \left(-i K_{2} l_{2}\right) & =b_{3}^{+}, \\
K_{2}\left[b_{2}^{+} \exp \left(i K_{2} l_{2}\right)-b_{2}^{-} \exp \left(-i K_{2} l_{2}\right)\right] & =K_{3} b_{3}^{+} .
\end{aligned}
$$

Here, $l_{1}$ is the thickness of substrate and $l_{2}$ is the thickness of PyC film. Equations (2) express continuity of tangential components of the electric and magnetic field on the vacuum/substrate, substrate/film, and film/vacuum interfaces. Reflectivity, transmittivity, and absorptivity of the $\mathrm{PyC}$ film on the silica can be obtained from the solution of Eq. (2) as

$$
R=\left|\frac{b_{0}^{-}}{b_{0}^{+}}\right|^{2}, \quad T=\left|\frac{b_{3}^{+}}{b_{0}^{+}}\right|^{2}, \quad A=1-R-T .
$$

The results obtained by the numerical solution of Eqs. (2) and (3) are presented in Fig. 3 by solid and dashed lines for $\mathrm{TE}_{1,0}$ waveguide mode. In the numerical calculations, we used the refractive index of substrate $n=3.6$ and the PyC film conductivity $\sigma=3.5 \times 10^{14} \mathrm{~s}^{-1}$, which was extracted from the experimental data ${ }^{8}$ and checked by fitting the theoretical absorption/transmission curves to the experimental ones collected in microwave frequency range (Fig. 3). It is worth noting that conductivity of the PyC film is lower than that of graphite at room temperature, $(0.6-1) \times 10^{16} \mathrm{~s}^{-1}$. This is because of scattering of the conduction electrons on the edges of small graphene ribbons that constitute the $\mathrm{PyC}$ film. Since in the $\mathrm{TE}_{1,0}$ mode the electric field is directed along the film surface, we used in the calculation the conductivity of the bulk PyC. In Fig. 3, one can see the excellent coincidence between experimental and numerical results for the absorption and transmission coefficients. In particular, both the experiment and numerical simulations demonstrate that PyC films with thicknesses of 30 and $75 \mathrm{~nm}$ absorb about $33 \%$ and $50 \%$ of the incident power, respectively (skin depth $\delta=c / \sqrt{2 \pi \sigma \omega}$ is about $15 \mu \mathrm{m})$.

Since the absorption losses are mainly due to the presence of the PyC film, it is instructive to consider dependence of the absorptivity on the film thickness when no substrate is involved. The reflectivity and transmittivity of a thin film in waveguide obtained from boundary conditions for tangential electric (magnetic) field components continuity can be presented in the following form:

$$
\begin{gathered}
R=\left|\frac{\left(K_{0}^{2}-K^{2}\right)[\exp (i K l)-\exp (-i K l)]}{\Delta}\right|^{2}, \\
T=\left|\frac{4 K_{0} K}{\Delta}\right|^{2},
\end{gathered}
$$

where $\Delta=\left(K_{0}-K\right)^{2} \exp (i K l)-\left(K_{0}+K\right)^{2} \exp (-i K l)$, for the $\mathrm{TE}_{1,0}$ mode $K_{0}=\sqrt{k^{2}-(\pi / a)^{2}}, K=\sqrt{k^{2} \varepsilon-(\pi / a)^{2}}$, 
where $\varepsilon$ is complex dielectric constant of the film. For the thin films $(|K l| \ll 1)$, Eqs. (4) and (5) yield

$$
R=\frac{1}{\left(1+l_{\sigma} / l\right)^{2}}, \quad T=\frac{1}{\left(1+l / l_{\sigma}\right)^{2}}, \quad A=\frac{2 l / l_{\sigma}}{\left(1+l / l_{\sigma}\right)^{2}} .
$$

Here, $l_{\sigma}=K_{0} c /(2 \pi k \sigma)$, where $\sigma$ is conductivity. It follows from Eq. (6) that at $l=l_{\sigma} A=0.5$, i.e., $50 \%$ of the EM radiation will be absorbed. It is important that $l_{\sigma}$ is much smaller than the skin depth.

In other words, for any material having metal-like conductivity there exists an optimal thickness, at which the film absorbs 50\% of the incident energy. This optimal thickness is inversely proportional to conductivity of the material. In the microwave frequency range, the optimal thickness is considerably less than the skin depth and does not exceed few nanometers for typical metals. For instance, the optimal thickness of the aluminium film with conductivity of $3.7 \times$ $10^{17} \mathrm{~s}^{-1}$ was observed experimentally as $3 \mathrm{~nm} .^{5}$

It is worth noting that in free space, $K_{0}=k$ (see Ref. 4) and hence the optimal thickness $l_{\sigma}$ is independent on the frequency, while the optimal thickness of the film in the waveguide is frequency dependent. In our particular case, $l_{\sigma}=91 \mathrm{~nm}$ at the frequency of $28 \mathrm{GHz}$.

In conclusion, we demonstrated that in the microwave frequency range, PyC films with thickness as small as few thousandth of the skin depth can absorb up to $50 \%$ of the incident power. PyC films have exceptional advantage of being hundred million times thinner than the thinnest nanocarbon based polymer coatings and almost 50000 times lighter than epoxy filled with carbon nanotubes. The later is especially important for satellite communication and other aerospace applications, where the ultra-light weight and ultra-small thickness are crucial. Moreover, chemically inert and corrosion resistant semitransparent $\mathrm{PyC}$ films can be deposited on both dielectric and metal substrate of any shape, opening avenues to development of advanced EM coatings, shields, and filters.

The work was partially supported by the EU FP7 projects FP7-266529 BY-NanoERA and CACOMEL FP7247007 as well BRFFI project F11 arm-006.

${ }^{1}$ S. Ramo and J. R. Whinnery, Fields and Waves in Communication Electronics, 3rd ed. (Wiley, New York, 1994).

${ }^{2} \mathrm{H}$. Ott, Electromagnetic Compatibility Engineering (John Wiley \& Sons, New York, 2009).

${ }^{3}$ G. E. H. Reuter and E. H. Sondheimer, Proc. R. Soc. London, Ser. A 195, 336 (1948).

${ }^{4}$ H. Bosman, Y. Y. Lau, and R. M. Gilgenbach, Appl. Phys. Lett. 82, 1353 (2003).

${ }^{5}$ V. G. Andreev, V. A. Vdovin, and P. S. Voronov, Tech. Phys. Lett. 29(11), 953 (2003).

${ }^{6}$ A. A. Neuber, L. Laurent, Y. Y. Lau, and H. Krompholz, in High-Power Microwave Sources and Technologies, edited by R. J. Barker and E. Schamiloglu (IEEE, New York, 2001).

${ }^{7}$ P. Kuzhir, A. Paddubskaya, S. Maksimenko, T. Kaplas, and Y. Svirko, Nanoscale Res. Lett. 8, 60 (2013).

${ }^{8}$ T. Kaplas and Y. Svirko, J. Nanophotonics 6, 061703 (2012).

${ }^{9}$ S. De and J. N. Coleman, ACS Nano 4, 2713 (2010).

${ }^{10}$ A. A. Green and M. C. Hersam, Nano Lett. 9, 4031 (2009).

${ }^{11}$ F. Qin and C. Brosseau, J. Appl. Phys. 111, 061301 (2012).

${ }^{12}$ V. Benzinge and A. Becker, Carbon 34, 957 (1996).

${ }^{13}$ A. C. Ferrari and J. Robertson, Philos. Trans. R. Soc. London, Ser. A 362, 2477 (2004).

${ }^{14}$ W. S. Bacsa, J. S. Lannin, D. L. Pappas, and J. j. Cuomo, Phys. Rev. B 47, 10931 (1993).

${ }^{15}$ T. Jawhari, A. Roid, and J. Casado, Carbon 33, 1561 (1995).

${ }^{16}$ A. S. Ferrari, Solid State Commun. 143, 47 (2007).

${ }^{17}$ Y. Wang, D. C. Alsmeyer, and R. L. McCreery, Chem. Mater. 2, 557 (1990).

${ }^{18}$ H. M. Kim, K. Kim, C. Y. Lee, J. Joo, S. J. Cho, H. S. Yoon, D. A. Pejaković, J. W. Yoó, and A. J. Epstein, Appl. Phys. Lett. 84, 589 (2004). 